\title{
Arbor
}

\section{Tecnologías emergentes para la conservación de alimentos sin calor}

\author{
Juan José Fernández-Molina, Gustavo V. \\ Barbosa-Cánovas y Barry G. Swanson
}

Arbor CLXVIII, 661 (Enero 2001), 155-170 pp.

Se han investigado los principios básicos de tres tecnologías emergentes para pasteurizar y esterilizar alimentos sin empleo del calor. Mediante numerosos estudios se ha comprobado la efectividad de los campos eléctricos pulsantes de alta intensidad (CEPAI), los pulsos de luz (PL) y los campos magnéticos oscilantes (CMO) en la destrucción de microorganismos y enzimas de sistemas alimentarios. En la inactivación microbiana por CEPAI, el blanco principal es la membrana celular que, al ser sometida a campos eléctricos de alta intensidad, se hace permeable formando huecos o poros cuyo tamaño se incrementa a medida que aumenta la intensidad del campo eléctrico o el tiempo de tratamiento o se reduce la resistencia iónica del medio de pulsación. Por otra parte, los PL inducen reacciones fotoquímicas y fototérmicas en los alimentos, causando la muerte de gran cantidad de microorganismos, especialmente en productos alimenticios envasados. Los CMO producen simulación o inhibición en el crecimiento y reproducción de los microorganismos, un simple pulso de intensidad de 5-10 teslas y frecuencias de 5-500 $\mathrm{kHz}$ es suficiente para reducir el número de microorganismos en un mínimo de 2 ciclos logarítmicos. Se ha comprobado que estas tecnologías alargan la vida de anaquel de diversos productos alimenticios y pueden ser consideradas como sustitutos parciales de los procesos convencionales de pasteurización y esterilización de alimentos por tratamientos térmicos. 
Fernández-Molina, Barbosa-Cánovas y Swanson

\section{Introducción}

La tecnología de conservación de alimentos se inició con el descubrimiento de Nicolás Appert a principios del siglo XIX. Este confitero francés, con el estímulo de un premio ofrecido por Napoleón a quien encontrara un procedimiento para conservar los alimentos para sus campañas militares, desarrolló un método que consistía esencialmente en envasarlos en botellas de vidrio cerradas y someterlos a cocciones de larga duración. De esta forma se conseguía almacenar diferentes alimentos por tiempos más o menos largos, según los casos, aunque la calidad de los productos así conservados dejaba bastante que desear. Con el tiempo este procedimiento empírico fue perfeccionándose hasta convertirse en una auténtica tecnología de conservación por calor, dando lugar a las conservas esterilizadas térmicamente que hoy conocemos, cuya conservación por largos períodos de tiempo se debe a la destrucción de los microorganismos responsables de su alteración y a la protección que los envases herméticamente cerrados le confieren frente a posibles recontaminaciones.

La eficacia de esta tecnología para conseguir la destrucción de los microorganismos que pueden alterar los alimentos durante su almacenamiento y de los patógenos, tiene como contrapartida que el calor aplicado produce alteraciones de la composición de los productos alimenticios. Estas alteraciones suponen en muchos casos pérdidas de calidad sensorial (los productos se reblandecen, cambia su sabor y su color) y de calidad nutritiva (pérdidas de vitaminas, principalmente). Aunque estas pérdidas son mínimas si el proceso térmico aplicado es correcto, los investigadores han continuado buscando alternativas de conservación de alimentos evitando el uso del calor, con el fin de conseguir alimentos más parecidos a los frescos en sus características nutritivas y sensoriales.

La esterilización de alimentos sin aplicación de calor, es decir por métodos no térmicos, constituye una alternativa novedosa de conservación de los alimentos. Las tecnologías que aparecieron al comienzo del siglo XX como prometedoras en la pasteurización de alimentos líquidos, como la leche, vienen a ser ahora tecnologías de avanzada, que ofrecen grandes ventajas en el procesamiento de alimentos sin aplicación de calor.

Los métodos eléctricos para pasteurizar y/o esterilizar alimentos están recibiendo gran atención en los últimos tiempos, debido al interés de la industria alimentaria en identificar métodos rápidos y uniformes de calentamiento o métodos de procesamiento a bajas temperaturas. 
Existen varios métodos para procesar alimentos a bajas temperaturas, entre los que figuran los calentamientos óhmicos y las microondas, los campos eléctricos, los campos magnéticos oscilantes, los arcos de descarga eléctrica y los campos eléctricos pulsantes de alta intensidad. La energía eléctrica puede ser aplicada al alimento en forma continua, generando calor en éste y promoviendo la inactivación de los microorganismos por efecto térmico. Por otra parte, si la energía es aplicada en forma de pulsos eléctricos cortos de alta intensidad, se generará muy poco calor en el alimento y la inactivación microbiana se logra con la destrucción de la membrana celular.

En este trabajo se hace una revisión de las nuevas alternativas tecnológicas que podrán, en un corto o mediano plazo, reemplazar parcialmente los tratamientos térmicos convencionales existentes, utilizados industrialmente para pasteurizar y/o esterilizar los alimentos. Se tratarán los campos eléctricos pulsantes de alta intensidad (CEPAI), los pulsos de luz (PL) y los campos magnéticos oscilantes (CMO), así como sus mecanismos de inactivación microbiana y la validez de estos procesos.

\section{Campos Eléctricos Pulsantes de Alta Intensidad (CEPAI)}

Los CEPAI constituyen una de las tecnologías más prometedoras para la conservación de los alimentos. La pasteurización con CEPAI involucra la utilización de pulsos eléctricos de alto voltaje en el alimento colocado entre dos electrodos. El tratamiento se realiza a temperatura ambiente o por debajo de ésta, en milésimas de segundos, y las pérdidas de energía por calor son minimizadas. Esta tecnología es considerada superior al tratamiento térmico convencional, debido a que reduce grandemente los cambios que ocurren en las propiedades sensoriales (sabor, color), y físicas (textura, viscosidad) de los alimentos (Quass, 1997). Además de conservar los atributos sensoriales de los alimentos, los CEPAI no introducen cambios químicos significativos en ellos y puede que no sean considerados como aditivo alimentario. Por el contrario, es una tecnología efectiva, segura y limpia.

Los aspectos más importantes de esta tecnología son la generación de campos eléctricos pulsantes de alta intensidad, el diseño de cámaras para el tratamiento del alimento, de tal manera que éste reciba un tratamiento uniforme con un mínimo incremento de la temperatura, y el buen diseño de electrodos para minimizar la electrólisis. Para generar los campos eléctricos de alta intensidad se utiliza un banco 
de condensadores conteniendo más de un condensador. Gran cantidad de esta energía se almacena en los condensadores mediante la carga de una fuente eléctrica de corriente alterna, el voltaje es entonces suministrado en forma de pulsos en la medida que el condensador es descargado (Zhang y col., 1995).

La aplicación de los CEPAI está restringida a aquellos productos alimenticios que puedan soportar campos eléctricos de alta intensidad. La constante dieléctrica del alimento está estrechamente relacionada con su estructura física y su composición química. Los líquidos homogéneos de baja conductividad eléctrica proporcionan las condiciones ideales para el tratamiento continuo con CEPAI. Los alimentos sólidos también pueden ser procesados con CEPAI en operaciones por lotes, siempre y cuando se evite la ruptura dieléctrica en el alimento. Las burbujas de aire en el fluido alimentario deben ser eliminadas cuando se usa este método, ya que, como soportan campos eléctricos de alta intensidad, causan arcos eléctricos, que pueden dar lugar a daños en la cámara y en los electrodos. En general, esta tecnología no es recomendable para el tratamiento de alimentos sólidos que retengan burbujas de aire al ser colocados en la cámara de tratamiento. Otra limitación es el tamaño de partícula de los alimentos sólidos. Para mantener una operación de proceso adecuada, el tamaño máximo de partícula en el fluido alimentario debe ser menor que la abertura de la región de tratamiento dentro de la cámara.

\section{Mecanismos de Inactivación Microbiológica por medio de Campos Eléctricos Pulsantes de Alta Intensidad}

Se han propuesto varias teorías para explicar la inactivación de los microorganismos con CEPAI. Las más estudiadas son la ruptura dieléctrica y la electroporación o desprendimiento de la membranas celulares (Zimermmann y Benz, 1980; 1986; Castro y col., 1993; Sale y Hamilton, 1967; Vega Mercado y col., 1996a, b). La aplicación de campos eléctricos a células biológicas en un medio (p.e., agua) causa la formación de cargas eléctricas en la membrana celular (Schoenbach y col., 1997). La destrucción de la membrana ocurre cuando el potencial eléctrico inducido en la membrana de muchos sistemas celulares excede el valor crítico de 1 voltio, lo que corresponde a un campo eléctrico externo de aproximadamente $10 \mathrm{kV} / \mathrm{cm}$ para la bacteria Escherichia coli (Castro y col., 1993). 
Tecnologías para la conservación...

FIGURA 1. Representación esquemática de la ruptura reversible de la membrana cecular

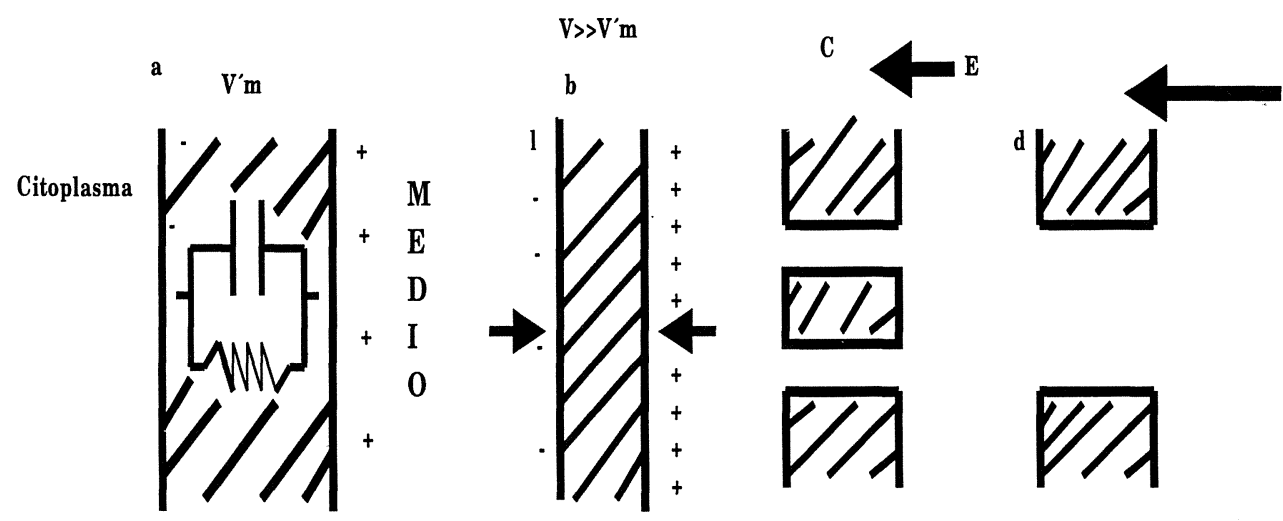

(a) membrana celular con potencial V'm, (b) compresión de la membrana, (c) formación de poros con ruptura reversible, (d) gran parte de la membrana sometida a ruptura irreversible con poros muy grandes.

Como se muestra en la Figura 1, Zimmermann (1986) explica el mecanismo de ruptura eléctrica de la membrana celular. La membrana puede ser considerada como un condensador lleno con circuito eléctrico (Fig. 1a). El potencial eléctrico normal en ambos lados de la membrana es de aproximadamente $10 \mathrm{mV}$. La exposición de la membrana (célula) a un campo eléctrico (pulsos) como se muestra en la Fig. 1b conduce al desarrollo en ésta de un potencial diferencial (V), promovido por la separación de cargas eléctricas a través de la membrana celular. V es proporcional al campo eléctrico (E) y al radio de la membrana. El aumento del potencial eléctrico de la membrana conlleva una reducción del espesor de la membrana celular. La ruptura de la membrana celular ocurre si el voltaje de ruptura crítico, $\mathrm{V}_{\mathrm{c}}(\sim 1 \mathrm{~V})$ es alcanzado por un incremento adicional del campo eléctrico externo, E (Fig. 1c). Se supone que la ruptura es causada por la formación de poros transmembránicos (llenados con solución conductora), lo cual conduce a una descarga inmediata de la membrana y a la descomposición de ésta. La ruptura es irreversible si los poros del producto son pequeños en relación con la superficie total de la membrana. A intensidades de campo eléctrico supercríticas y largos tiempos de exposición, grandes áreas de la membrana estarán sujetas a la ruptura (Fig. 1d). Si el tamaño y el número de poros se hace mayor, en relación con la superficie total de la membrana, la ruptura reversible cambia a una ruptura irreversible, la cual está asociada con la destrucción mecánica de la membrana celular. 
El otro mecanismo propuesto, la electroporación, es un fenómeno que desestabiliza temporalmente la capa lipídica y las proteínas de la membrana celular, al ser sometidas a campos eléctricos pulsantes de alto voltaje (Castro y col., 1993). El plasma de las membranas celulares se hace permeable a pequeñas moléculas, después de haber sido expuesto a un campo eléctrico, y la permeabilidad causa hinchazón y una eventual ruptura de la membrana celular como se muestra en la Fig. 2 (Vega-Mercado y col., 1996b). El principal efecto de los campos eléctricos en la membrana celular es, por lo tanto, causar permeabilidad debido a la compresión y formación de poros en ésta (Vega-Mercado et al., 1996b). Kinosita y Tsong (1977a, 1979) demostraron que un campo eléctrico de $2.2 \mathrm{kV} / \mathrm{cm}$ inducía poros en eritrocitos humanos de aproximadamente $1 \mathrm{~mm}$ de diámetro (Martín y col., 1995). Kinosita y Tsong (1977a) sugirieron un mecanismo de dos pasos para la formación de los poros, en el cual el inicial es una respuesta a un potencial de campo eléctrico superior al umbral, seguido de una expansión del tamaño del poro en el tiempo, como se indica en la Fig. 2. Se obtienen grandes poros mediante incrementos de la intensidad del campo eléctrico y la duración del pulso o reduciendo la resistencia iónica del medio de pulsación. En la membrana celular las cargas eléctricas bipolares de los lípidos, proteínas, carbohidratos, iones, y la polarizabilidad de estas moléculas forman el campo eléctrico. Por lo tanto la electroporación ocurre en los liposomas y en las membranas celulares, pero las moléculas afectadas por el campo aplicado no son necesariamente las mismas en estos dos sistemas (Tsong, 1990).

FIGURA 2. Electroporación de una membrana celular mostrando las zonas de hinchamiento, lisis e inactivación celular

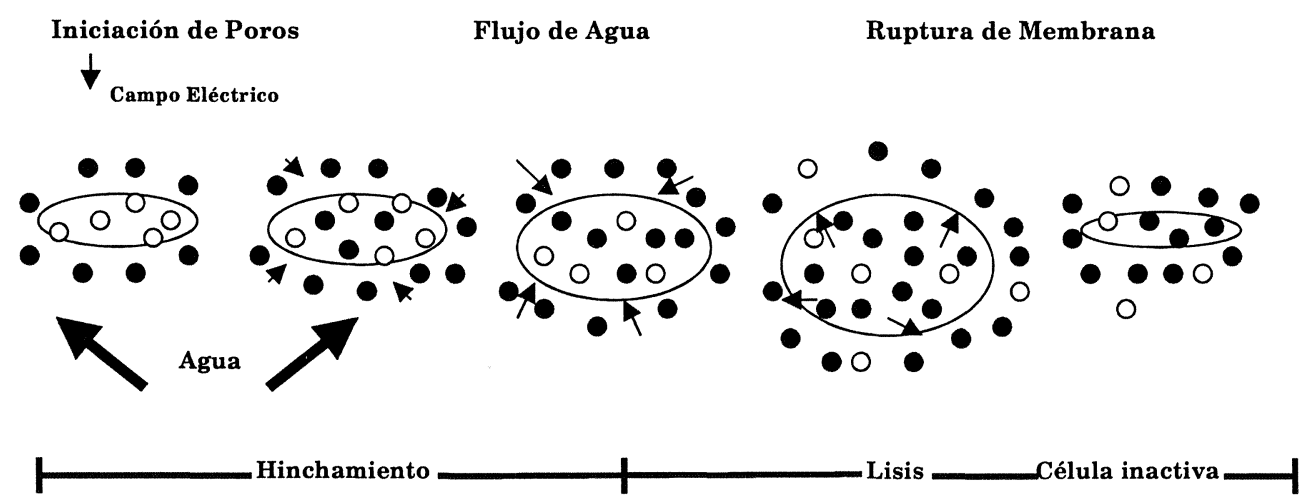


En una vesícula lipídica, los movimientos electroforéticos de iones y dipolos de agua a través de poros hidrofóbicos espontáneos pueden ser el primer paso de la electroporación; después de lo cual las moléculas se reorganizan para formar poros hidrofílicos estables. En una membrana celular esto puede ocurrir, sin embargo, también están presentes canales proteicos, poros y bombas. Estas membranas son extremadamente sensibles al campo eléctrico transmembránico (Tsong, 1990). Los potenciales de abertura a los canales constituidos por las proteínas están en el orden de los $50 \mathrm{mV}$ (Castro y col., 1993).

Miller y col. (1988) encontraron que la electroporación permite la toma de $\mathrm{ADN}$ por las células mamarias y protoplastos de las plantas, debido a que ésta reduce la permeabilidad de la membrana celular. Estos investigadores demostraron la utilidad de la electroporación de altos voltajes para la transformación genética de células bacterianas intactas, utilizando la bacteria patógena Campylobacter jejuni como sistema modelo. El método involucra la exposición de células en suspensión de Campylobacter a un potencial exponencial de alto voltaje con una descarga de $5-13 \mathrm{kV} / \mathrm{cm}$ con tiempos de tratamiento cortos de 2,4-2,6 s en la presencia de ADN plásmido.

\section{Validación del Proceso de Campos Eléctricos Pulsantes de Alta Intensidad}

Se han realizado pruebas extensivas de inactivación microbiana para validar el concepto de CEPAI como un proceso de pasteurización de alimentos sin calor (Zhang y col., 1994; Zhang y col. 1995; Pothakamury y col., 1995; Keith y col., 1997; Marquez y col., 1997; Qin y col., 1995; Vega-Mercado y col., 1996a, b; Qin y col., 1998; Castro y col., 1993).

Los campos eléctricos pulsantes de alta intensidad producen una serie de cambios degradantes en las células de sangre, algas, bacterias y levaduras (Castro y col., 1993). Estos cambios incluyen electroporación y desprendimiento de las membranas semipermeables, lo que conduce al hinchamiento y/o encogimiento y finalmente lisis de la célula. Los mecanismos de inactivación de microorganismos incluyen ruptura eléctrica, efecto iónico de pulso y electroporación de las membranas celulares (Vega-Mercado y col., 1996b).

La inactivación microbiana usando CEPAI, reportada por varios investigadores se resume en la tabla 1. En las citas contenidas en esta tabla puede encontrarse información detallada sobre los tratamientos aplicados y los resultados obtenidos. 
Fernández-Molina, Barbosa-Cánovas y Swanson

TABLA 1. Resumen de inactivaciones microbiológicas mediante el uso de campos eléctricos pulsantes de alta intensidad

\begin{tabular}{|c|c|c|c|c|c|c|c|}
\hline Referencias & $\begin{array}{c}\text { Microorganismos } \\
\text { e Inoculación } \\
\text { inicial }\end{array}$ & $\begin{array}{l}\text { Medio de } \\
\text { suspensión }\end{array}$ & $\begin{array}{l}\text { Reducción } \\
\text { logarítmica } \\
(\mathrm{ufc} / \mathrm{mL})\end{array}$ & $\begin{array}{l}\text { Tempe- } \\
\text { ratura } \\
{ }^{\circ} \mathrm{C}\end{array}$ & $\begin{array}{r}\text { Campo } \\
\text { eléctrico } \\
(\mathrm{kV} / \mathrm{cm})\end{array}$ & $\begin{array}{l}\text { Tiempo } \\
(\mu \mathrm{s})\end{array}$ & $\begin{array}{c}\text { Número } \\
\text { de } \\
\text { pulsos }\end{array}$ \\
\hline Martín y col., 1997 & E. coli $\left(10^{9} \mathrm{cfu} / \mathrm{ml}\right)$ & Leche Descremada & $1-3$ & 15 & $20-45$ & $0,7-1,8$ & 64 \\
\hline Márquez y col., 1997 & $\begin{array}{l}\text { B. subtilis } \\
3 \times 10^{3} \text { spores } / \mathrm{ml} \\
\text { B. cereus } \\
10^{5} \text { sporos } / \mathrm{ml}\end{array}$ & $\begin{array}{l}0,15 \% \mathrm{NaCl} \\
0,15 \% \mathrm{NaCl}\end{array}$ & $\begin{array}{c}3.425 \\
5\end{array}$ & $\begin{array}{l}25 \\
25\end{array}$ & $\begin{array}{l}50 \\
50\end{array}$ & $\begin{array}{l}2-3 \\
5-6\end{array}$ & $\begin{array}{l}30 \\
50\end{array}$ \\
\hline Zhang y col., 1995 & E. coli $\left(10^{9} \mathrm{cfu} / \mathrm{ml}\right)$ & Leche Modificada & 9 & 20 & 70 & 160 & 80 \\
\hline $\begin{array}{l}\text { Hülshegher y } \\
\text { col., } 1983\end{array}$ & E. coli & Buffer Fosfato & 4 & 20 & 20 & 1,080 & 30 \\
\hline Grahl y col., 1993 & E. coli $\left(10^{7}\right)$ & Leche & 3 & - & 22 & 200 & 5 \\
\hline Pagán y col., 1997 & B. subtilis & Leche Modificada & 5 & 60 & $60+$ HHP $*$ & - & 75 \\
\hline $\begin{array}{l}\text { Pothakamury y } \\
\text { col., } 1995\end{array}$ & $\begin{array}{l}\text { E. coli } \\
\text { S. aureus }\end{array}$ & Leche Modificada & $\begin{array}{c}3-4 \\
2\end{array}$ & $\begin{array}{l}37 \\
37\end{array}$ & $\begin{array}{l}16 \\
16\end{array}$ & $\begin{array}{l}200-300 \\
200-300\end{array}$ & $\begin{array}{l}60 \\
60\end{array}$ \\
\hline $\begin{array}{l}\text { Pothakamury } \\
\text { et al., 1995b }\end{array}$ & $\begin{array}{l}\text { B. subtilis, and L. } \\
\text { delbrueckii }\end{array}$ & Leche Modificada & $4-5$ & 30 & 16 & $200-300$ & $40-50$ \\
\hline $\begin{array}{l}\text { Vega-Mercado y } \\
\text { col., } 1996\end{array}$ & E. coli $\left(10^{7}\right)$ & Leche Modificada & 2,2 & 10 & $40-50$ & - & 8 \\
\hline Qin y col., 1988 & S. cerevisiae & Jugo de Manzana & $>6$ & $>40$ & 36 & - & - \\
\hline Raso y col., 1988 & $\begin{array}{l}\text { Zygosaccharomyces } \\
\text { bailii }\end{array}$ & $\begin{array}{l}\text { Jugos de naranja, } \\
\text { uva, fresas }\end{array}$ & 5 & 20 & $32-36,5$ & $2-3,5$ & - \\
\hline Keith y col., 1997 & Microorganismos & Especias & 1 & - & $20-80$ & $1-99$ & $11-28$ \\
\hline Sensoy y col., 1997 & $\begin{array}{l}\text { Salmonella dublin } \\
\left(10^{5}\right)\end{array}$ & $\begin{array}{l}\text { Leche descremada, } \\
\mathrm{KCl}\end{array}$ & 3 & $10-50$ & $15-40$ & $12-127$ & - \\
\hline
\end{tabular}

* HHP: Alta presión hidrostática

El proceso de tratamiento con CEPAI ha demostrado que extiende la vida de anaquel de los jugos de manzana hasta cuatro semanas, diez días para jugos frescos de naranja y hasta dos semanas para leche cruda descremada. Qin y col. (1995) no encontraron diferencias significativas en la evaluación sensorial de huevos frescos líquidos y tratados con CEPAI. Más aún, los huevos tratados con CEPAI fueron preferidos por el panel de catadores frente a los huevos líquidos, provenientes de huevos líquidos comerciales. Barbosa-Cánovas y col. (1998) reportaron que el tratamiento de CEPAI en crema de guisantes extendía la vida de anaquel hasta cuatro semanas a $4^{\circ} \mathrm{C}$. Las propiedades químicas, físicas y sensoriales de esta crema no variaron después del 
tratamiento con CEPAI y después del almacenamiento. Dunn y Pearlman (1987) encontraron que la vida de anaquel de yogur, inoculado con $S$. cerevisiae y tratado con CEPAI a $45^{\circ} \mathrm{C}$, podía aumentarse hasta diez días cuando se almacenaba a $4^{\circ} \mathrm{C}$. Aumentando el tratamiento con CEPAI y la temperatura a $55^{\circ} \mathrm{C}$ se extendía la vida de anaquel del yogur hasta un mes, almacenado a $4^{\circ} \mathrm{C}$.

\section{Tecnología de Pulsos de Luz (PL)}

Los pulsos de luz son producidos utilizando tecnologías de ingeniería que multiplican la potencia varias veces. La potencia se magnifica por la acumulación de energía eléctrica en un condensador que almacena energía por tiempos relativamente largos (fracciones de segundos). Esta energía almacenada se utiliza para realizar el trabajo en tiempos mucho más cortos (millones o miles de segundos). El resultado es una potencia elevada durante el ciclo de trabajo, con un gasto moderado en el consumo de energía (Dunn, 1996).

Las tecnologías de Pure Pulse han desarrollado dos nuevos procesos para matar microorganismos, asociados con el envasado de productos alimenticios, suministros médicos, farmacéuticos, agua y aire (Dunn, 1996). Estos nuevos procesos son denominados por su nombre en inglés «Pure Bright» $\mathrm{y}$ «Cool Pure».

Los pulsos de luz denominados «Pure Bright» utilizan rayos de luz de corta duración en el espectro amplio de luz blanca para matar un amplio número de microorganismos incluyendo esporos y hongos. Cada pulso de luz dura solamente millonésimas de segundos. Durante cada pulso que pasa la intensidad de la luz es de unas 200.000 veces la intensidad de la luz en la superficie terrestre (Dunn, 1996). El proceso de campos eléctricos pulsantes de alta intensidad (Cool Pure) utiliza múltiples pulsos de corta duración, y campos eléctricos pulsantes de alta intensidad para inactivar los microorganismos en los alimentos transportados por tuberías. El intervalo de temperaturas durante la aplicación de este proceso es muy bajo, por lo que no causa daños térmicos apreciables. El sabor original, textura y funcionalidad de los productos alimenticios se mantiene (Dunn, 1996).

\section{Mecanismos de Inactivación con Pulsos de Luz (PL)}

La letalidad de los pulsos de luz es diferente a distintas longitudes de ondas. Por lo tanto, para tratar los alimentos se puede utilizar el 
espectro completo o la longitud de onda seleccionada. Las longitudes de onda conocidas que producen productos indeseables en los alimentos se eliminan a través de filtros de vidrio o filtros líquidos. Los pulsos de luz inducen reacciones fotoquímicas o fototérmicas en el alimento. La luz ultravioleta causa cambios fotoquímicos mientras que la luz visible e infrarroja causan cambios fototérmicos. Los efectos antimicrobianos de estas longitudes de ondas son primariamente mediados a través de la absorción de sistemas conjugados de dobles enlaces carbono-carbono en proteínas y ácidos nucleicos.

El material a esterilizar se expone como mínimo a un pulso de luz con una densidad de energía en el intervalo de 0,01 a $50 \mathrm{~J} / \mathrm{cm}^{2}$ en la superficie, usando una distribución de longitudes de onda, de tal manera que por lo menos un $70 \%$ de la energía electromagnética se distribuya en un intervalo de longitudes de onda de 170 a 2600 nm (Barbosa-Cánovas y col., 1997). La duración de los pulsos varía entre 1 y $0,01 \mu \mathrm{s}$. Los rayos se aplican a una tasa de 1 a 20 rayos por segundo. Para la mayoría de las aplicaciones, pocos rayos aplicados en fracciones de segundo suministran un alto nivel de inactivación microbiana. Por lo tanto el proceso es muy rápido y sencillo para la obtención de altos rendimientos.

\section{Validación del Proceso de Pulsos de Luz (PL)}

Los pulsos de luz proporcionan una extraordinaria ampliación de la vida de anaquel de una gran variedad de alimentos. Se ha comprobado que el proceso es efectivo en la inactivación de hongos en gran variedad de productos horneados. Camarones tratados con PL, almacenados y refrigerados permanecieron comestibles por siete días, mientras que los no tratados mostraron una notable degradación, decoloración, olores desagradables y no eran comestibles (Dunn y col., 1995). Barbosa-Cánovas y col. (1998) reportaron más de siete ciclos logarítmicos de reducción en la inactivación de esporas de Aspergillus niger tratados con PL. Una gran variedad de microorganismos, incluyendo $E$. coli, $S$. aureus, $B$. subtilis y $S$. cerevisiae, se inactivaron utilizando 1 a 35 pulsos de luz con una intensidad entre 1 y $2 \mathrm{~J} / \mathrm{cm}^{2}$. Con el proceso «Pure Bright» de PL la Salmonella pudo ser reducida, en dos ciclos logarítmicos, en muestras de alas de pollos inoculadas con 5 ó $2 \mathrm{log} / \mathrm{cm}^{2}$. La listeria también fue reducida en dos ciclos logarítmicos en salchichas (inoculadas con 3 ó $5 \mathrm{log} / \mathrm{salchicha)} \mathrm{después} \mathrm{del} \mathrm{tratamiento} \mathrm{con} \mathrm{PL}$ (Barbosa-Cánovas y col., 1998). 
Tecnologías para la conservación...

El tratamiento con PL de cuajadas comerciales de queso seco (cottage), inoculadas con Pseudomonas, con una densidad de energía de $16 \mathrm{~J} / \mathrm{cm}^{2}$ y una duración de pulso de $0,05 \mathrm{~ms}$, redujo la población microbiana en 1,5 ciclos logarítmicos después de la aplicación de dos rayos de luz. La temperatura en la superficie de la cuajada estaba cercana a la fuente de luz y aumentó $5^{\circ} \mathrm{C}$ (Dunn y col., 1991). Un panel de evaluación sensorial entrenado demostró que no se habían producido efectos en el sabor de los quesos tratados con PL. Una combinación de alta presión y exposición a PL redujo la población de coliformes de la superficie de tejidos de pescados en tres ciclos logarítmicos. Las muestras de pescado permanecieron sensorialmente aceptables después de quince días de almacenadas en refrigeración (Dunn y col., 1988).

Los pulsos de luz han sido muy efectivos en la eliminación de la contaminación microbiana de la superficie de cáscaras de huevo. Se han conseguido hasta ocho ciclos logarítmicos de reducción, sin encontrar diferencias entre huevos comerciales y huevos crudos. En la superficie de diferentes materiales de envasado un simple pulso de luz inactivó $S$. aureus con una cantidad de energía tan pequeña como $1,25 \mathrm{~J} / \mathrm{cm}^{2}$ mientras que esporas de $B$. cereus y Apergillus fueron inactivadas con una densidad de energía superior a $2 \mathrm{~J} / \mathrm{cm}^{2}$ (Barbosa-Cánovas y col., 1998).

\section{Campos magnéticos oscilantes}

La utilización de campos magnéticos oscilantes para la inactivación de microorganismos tiene el potencial de pasteurizar alimentos con una mejora en la calidad y en la vida de anaquel, en comparación con los procesos convencionales de pasteurización. Los campos magnéticos pueden ser estáticos (CMS) u oscilantes (CMO). En el campo magnético estático la intensidad del campo magnético es constante con el tiempo, mientras que en un campo magnético oscilante se aplica en forma de pulsos. La carga de los pulsos es inversa en cada pulso. El campo magnético puede ser homogéneo o heterogéneo. En un campo magnético homogéneo la intensidad del campo (B) es uniforme en el área envuelta por el campo magnético mientras que en un campo magnético heterogéneo, $\mathrm{B}$ no es uniforme con las intensidades, disminuyendo así como las distancias del centro del alambre aumentan. Los campos magnéticos oscilantes, aplicados en forma de pulsos, invierten la carga en cada pulso pero también la intensidad de cada 
pulso disminuye con el tiempo en un $10 \%$ de la intensidad inicial (Pothakamury y col., 1993 ).

La exposición a los campos magnéticos causa estimulación o inhibición en el crecimiento y reproducción de los microorganismos. Un simple pulso de intensidad de 5 a 10 tesla (T) y frecuencia de 5 a $500 \mathrm{kHz}$ se aplica generalmente para reducir el número de microorganismos por lo mínimo en dos ciclos logarítmicos (Barbosa-Cánovas y col., 1996). Los campos magnéticos oscilantes de esta magnitud se pueden generar utilizando: (1) alambres superconductores; (2) alambres que producen campos DC; o (3) alambres energetizados por la descarga de energía almacenada en un condensador (Barbosa-Cánovas y col., 1998). La inhibición o estimulación de microorganismos, expuestos a campos magnéticos, puede ser el resultado de un campo magnético o de campos eléctricos inducidos. Este último es medido en términos de la intensidad del campo eléctrico inducido y la densidad de corriente inducida. Para diferenciar entre efectos de campos magnéticos y campos eléctricos se recomienda el uso de un cilindro que contiene células y un medio que pueda ser adaptado a estudios in vitro, empleando campos magnéticos uniformes de fases sencillas y frecuencias extremadamente bajas.

\section{Mecanismos de Inactivación Microbiana con Campos Magnéticos Oscilantes}

Pothakamury y col. (1993) reportaron dos teorías que explican los mecanismos de inactivación de células colocadas en campos magnéticos estáticos u oscilantes. La primera teoría afirma que un campo magnético oscilante débil puede debilitar los enlaces entre iones y proteínas. Muchas proteínas vitales para un metabolismo saludable contienen iones. En presencia de un campo magnético inmóvil, como el de la tierra, los efectos biológicos de los campos magnéticos oscilantes son más pronunciados alrededor de frecuencias particulares, tales como la frecuencia de resonancia del ciclotrón de iones.

A una resonancia de ciclotrón, la energía es transferida selectivamente del campo magnético al ión con una girofrecuencia equivalente a la frecuencia del campo magnético. El sitio de interacción del campo magnético es el tejido de la célula, que viene a ser el más afectado por éste. Los iones transmiten los efectos de los campos magnéticos a otros tejidos de órganos a través del sitio de interacción.

Una segunda teoría considera el efecto de los campos magnéticos estáticos y oscilantes en enlaces de iones de calcio pegados a proteínas 
tales como el calmodulin. Los iones de calcio continuamente vibran alrededor de una posición de equilibrio en el sitio de enlace del calmodulin. Aplicando un campo magnético inmóvil al calmodulin causa rotación y vibración del plano o procede en la dirección del campo magnético a una frecuencia que es exactamente la frecuencia del ciclotrón del enlace del calcio. Al agregar un campo magnético vibratorio a la frecuencia del ciclotrón se perturba la precisión a tal extensión que resulta en la debilitación del enlace entre el ion de calcio y el calmodulin (Pothakamury y col., 1993). La inactivación de microorganismos está basada en la teoría de los campos magnéticos oscilantes, los cuales pueden acumular la energía en partes activamente magnetizadas de grandes moléculas como las de ADN. Dentro de un intervalo de 5 a $50 \mathrm{~T}$, la cantidad de energía por oscilaciones acopladas a un dipolo de ADN es de $10^{-2}$ a $10^{-3} \mathrm{eV}$. Con diferentes oscilaciones y ensamblaje colectivo de dipolos se obtiene suficiente activación local que puede resultar en la ruptura de los enlaces covalentes de la molécula de ADN y por consiguiente en la inactivación de los microorganismos (Pothakamury y col., 1993).

\section{Validación del Proceso de Campos Magnéticos Oscilantes (CMO)}

Hoffman (1985) investigó la validación del proceso de campos magnéticos oscilantes. Este autor reportó la inactivación de microorganismos con CMO con una densidad de flujo mayor a $2 \mathrm{~T}$ para obtener una reducción de, como mínimo, dos ciclos logarítmicos.

La conservación de alimentos con CMO involucra colocar los alimentos en bolsas plásticas selladas y someterlas de 1-100 pulsos con una frecuencia de 50 a $500 \mathrm{kHz}$ y temperatura de 0 a $50^{\circ} \mathrm{C}$ para un tiempo total de exposición que varia entre 25 y $100 \mathrm{~ms}$. Las frecuencias mayores de $500 \mathrm{kHz}$ son menos efectivas para la inactivación microbiana y tienden a calentar el alimento (Barbosa-Cánovas y col., 1998). Los tratamientos con campos magnéticos se llevan a cabo a presión atmosférica y a una temperatura que estabiliza el material alimenticio. El alimento es esterilizado sin cambios apreciables en su calidad y la temperatura del alimento aumenta entre 2 y $5{ }^{\circ} \mathrm{C}$. Hoffman (1985) ha reportado la inactivación de microorganismos en productos como la leche, yogur, jugo de naranja y pan, tratados con CMO. Los resultados indican que solamente un pulso de CMO es suficiente para reducir la población bacteriana entre $10^{2}$ y $10^{3}$ microorganismos/gramo. La intensidad del campo magnético, requerida para obtener estos efectos, varía entre 2-25 $\mathrm{T}$ y las frecuencias entre 5-500 kHz (Pothakamury y col., 1993). 


\section{Conclusión}

La aplicación de tecnologías alternas para pasteurizar y esterilizar alimentos sin calor, como los campos eléctricos pulsantes de alta intensidad, los pulsos de luz y los campos magnéticos oscilantes, constituyen un potencial a ser explotado por la industria alimentaria. Su forma única de aplicación a bajas temperaturas hace de estas tecnologías una alternativa de sustitución de los procesos térmicos tradicionales utilizados en la pasteurización y esterilización de alimentos líquidos.

Los resultados de estudios experimentales han demostrado la eficacia y validación de estos métodos en la conservación y extensión de la vida de anaquel de productos alimenticios como la leche, huevos líquidos, jugos de manzana, naranja y yogures, entre otros. Los campos eléctricos pulsantes de alta intensidad y los pulsos de luz son las dos tecnologías más estudiadas y que parecen estar listas para su aplicación industrial, como se ha demostrado en pruebas de laboratorio y de planta piloto.

\section{Bibliografía}

Barbosa-Cánovas, G.V., Góngora-Nieto, M.M., y Swanson, B.G. 1998. Nonthermal electrical methods in food preservation. Food Sci. Technol. Int. 4(5):363-370.

Barbosa-Cánovas, G.V., Palou, E., Pothakamury, U.R., y Swanson, B.G. 1997. Application of light pulses in the sterilization of foods and packaging materials. In: Nonthermal Preservation of Foods. Cap. 6, Marcell Dekker, New York.

Castro, A.J. (1994). Pulsed Electrical Field Modification of Activity and Denaturation of Alkaline Phosphatase. Ph.D. Dissertation. Washington State University-Food Science and Human Nutrition, Pullman, WA, USA.

Castro, A.J., Barbosa-Cánovas, G.V., y Swanson, B.G. (1993). Microbial inactivation of foods by pulsed electric fields. J. Food Process \& Preserv. 17:47-73.

DunN, J. 1996. Pulsed light and pulsed electric field for foods and eggs. Poultry Science. 75:1133-1136.

Dunn, J., Clark, R.W., Asmus, J.F., Pearlman, J.S., Boyer, K., Pairchaud, F., y Hofmann, G. 1988. Methods and apparatus for preservation of foodstuffs. U.S. Int. Pat. Appl. No. WO 88/03369.

Dunn, J., y Pearlman, J.S. 1987. Methods and apparatus for extending the shelf-life of fluid food products. U.S. Patent 4,695,472.

DunN, J., OTt, T., y Clark, R.W. 1995. Pulsed-light treatment of food packaging. Food Technology. 49(9):95-98.

Dunn, J., Clark, R.W., Asmus, J.F., Pearlman, J.S., Boyer, K., Pairchaud, F., y Hofman, G. 1991. Methods and apparatus for preservation of foodstuffs. U.S. Patent $5,034,235$.

Grahl, T., SitzmanN, W., y Markel, H. (1992). Killing of microorganisms in fluid media by high-voltage pulses. DECHEMA Biotechnol. Conference Series. 5B:675-8. 


\section{Tecnologías para la conservación...}

Ho, S.Y., MrtTal, G.S., y Cross, J.D. (1997). Effects of high field electric pulses on the activity of selected enzymes. J. Food Eng. 31:69-85.

Hofmann, G.A. 1985. Deactivation of microorganisms by an oscillating magnetic field. U.S. Patent 4,524,079.

Hülsheger, H., Pottel, J. y Niemann, E.G. (1983). Electric field effects on bacteria and yeast cells. Radiat. Environ. Biophys. 22:149-162.

Hülsheger, H., Pottel, J. y Niemann, E.G. (1981). Killing of bacteria with electric pulses of high field strength. Radiat. Environ. Biophys. 20:53-65.

KeIth, W.D., HarRiS, L.J., Hudson, L., y Griffiths, M.W. (1997). Pulsed electric fields as a processing alternative for microbial reduction in spice. J. Food Res. Intern. 30(3/4):185-191.

Kinosita, K., Jr. y Tsong, T.Y. (1977a). Voltage induced pore formation and hinemolysis erythrocytes. Biochim. Biophys. Acta. 471:227-242.

Kinosita, K., Jr. y Tsong, T.Y. (1979). Voltage-induced conductance in human erythrocyte membranes. Biochim. Biophys. Acta. 554:479-497.

Márquez, V.O., Mittal, G.S., y Griffiths, M.W. (1997). Destruction and inhibition of bacterial spores by high voltage pulsed electric fields. J. Food Sci. 62(2):399401.

Martin, O., Qin, B.L., Chang, F.J., Barbosa-Cánovas, G.V., y Swanson, B. 1997. Inactivation of Escherichia coli in skim milk by high intensity pulsed electric fields. J. Food Proc. Eng. 20:317-336.

Martin, O., Zhang, Q., Castro, A.J., Barbosa-Cánovas, G.V., y Swanson, B.G. (1995). Empleo de pulsos eléctricos de alto voltaje para la conservación de alimentos. Microbiología e ingeniería del proceso. Rev. Esp. Cienc. Tecnol. Aliment. 34(1):134.

Miller, J.F., Dower, W.J., y ToMPkins, L.S. (1988). High-voltage electroporation of bacteria: Generic transformation of Campylobacter jejuni with plasmid DNA. Proc. Natl. Acad. Sci. 85:856-860.

Pagán, R., Espulgas, S. Góngora-Nieto, M.M., Barbosa-Cánovas, G.V., y Swanson, B.G. (1997). Inactivation of Bacillus subtilis spores using high intensity pulsed electric fields in combination with other food conservation technologies. J. Food Sci. Technol. Intern. 4:33-44.

Pothakamury, U.R., Barbosa-Cánovas, G.V., y Swanson, B.G. (1993).Magnetic-field inactivation of microorganisms and generation of biological changes. Food Technology. 47(12):85-93.

Pothakamury, U.R., Barbosa-Cánovas, G.V., y Swanson, B.G. (1993). Inactivation of microorganisms by oscillating magnetic fields. Rev. Esp. Cienc. Tecnol. Aliment. 33(5):479-489.

Pothakamury, U.R., Monsalve-González, A., Barbosa-Cánovas, G.V., y Swanson, B.G. (1995). Inactivation of Escherichia coli and Staphylococcus aureus in model foods by pulsed electric field technology. J. Food Res. Intern. 28(2):167-171.

Pothakamury, U.R., Monsalve-González, A., Barbosa-Cánovas, G.V., y Swanson, B.G. (1995b). High voltage pulsed electric field inactivation of Bacillus subtilis and Lactobacillus delbrueckii. Rev. Esp. Cienc. Tecnol. Aliment. 35(1):101-107.

Qin, B., Pothakamury, U.R., Vega, H., Martin, O., Barbosa-Cánovas, G.V., y Swanson, B.G. (1995). Food pasteurization using high intensity pulsed electric fields. $J$. Food Technol. 49(12): 55-60. 
Qin, B., Barbosa-Cánovas, G.V., Swanson, B.G., y Pedrow, P.D. (1998). Inactivating microorganism using a pulsed electric field continuous treatment system. IEEE Trans. Indus. Applic. 34(1):43-49.

Quass, D.W. 1997. Pulsed electric field processing in the food industry. A status report. CR-109742. Electric Power Institute. Palo Alto California.

Raso, J. Calderón, M.L., Góngora-Nieto, M. Barbosa-Cánovas, G.V., y Swanson, B.G. 1998. Inactivation of Zygosaccharomyces bailii in fruit juices, high hydrostatic pressure and pulsed electric fields. J. Food Sci. 63(6):1042-1044).

REINA, L.D., Jin, Z.T., ZHANG, Q.H., y YouSEF, A.E. 1998. Inactivation of Listeria monocytogenes in milk by pulsed electric field. J. Food Prot. 61(9):1203-1206.

Sale, A.J., y Hamilton, W.A. (1967). Effect of high electric fields on microorganisms. I. Killing of bacteria and yeast. Biochim. Biophys. Acta. 148:781-788.

Sensoy, I., Zhang, Q.H., y SASTRY, S.K. (1997). Inactivation kinetic of Salmonella dublin by pulsed electric fields. J. Food Proc. Eng. 20:367-381.

Schoendach, K.H., Peterkin, F.E., Alden, R.W., y Beebe, S.J. (1997). The effect of pulsed electric fields on biological cells: Experiments and applications. IEEE Trans. Plasma Sci. 25(2):284-292.

Tsong, T.Y. (1990). Electrical modulation of membrane proteins: Enforced conformational oscillations and biological energy signals. Annu. Rev. Biophys. Chem. 19:83-106.

Vega-Mercado, H., Powers, R.J., Barbosa-Cánovas, G.V., y Swanson, B.G. (1995a). Plasmin inactivation with pulsed electric fields. J. Food Sci. 60:1143-1146.

Vega-Mercado, H., Martín-Belloso, O., Chang, F.J., Barbosa-Cánovas, G.V., y Swanson, B.G (1996a). Inactivation of Escherichia coli and Bacillus subtilis in pea soup using pulsed electric fields. J. Food Process \& Preserv. 20:501-510.

Vega-Mercado, H., Pothakamury, U.R., Chang, F.J., Barbosa-Cánovas, G.V., y Swanson, B.G (1996b). Inactivation of Escherichia coli by combining $\mathrm{pH}$, ionic strength and pulsed electric fields. J. Food Research International. 29(2): 117-121.

VEga-Mercado, H. (1996). Inactivation of Proteolytic Enzymes and Selected Microorganisms in Foods Using Pulsed Electric Fields. Ph.D. Dissertation. Washington State University-Biological Systems Engineering. Pullman, WA., USA.

Zhang, Q., Barbosa-CÁnovas, G.V., y Swanson, B.G. (1995). Engineering aspects of pulsed electric field pasteurization. J. Food Eng. 25:261-281.

Zhang, Q., Qin, B., Barbosa-Cánovas, G.V., y Swanson, B.G. (1994). Inactivation of E. coli for food pasteurization by high -strength pulsed electric fields. J. Food Proc. Preserv. 19:103-118.

ZimmermanN, U. (1986). Electric breakdown, electropermeabilization and electrofusion. Rev. Physiol. Biochem. Pharmacol. 105:175-256.

Zimmermann, U., y Benz, R. (1980). Dependence of the electrical breakdown voltage on the charging time in Valonia utricularis. J. Membr. Biol. 53:33-43. 